\title{
Intra-articular hyaluronic acid after knee arthroscopy: a two-year study
}

\author{
Harald Hempfling
}

Received: 2 February 2006/ Accepted: 20 November 2006/Published online: 23 December 2006

(C) Springer-Verlag 2006

\begin{abstract}
Arthroscopic knee joint lavage is used when conservative treatment of knee osteoarthritis is unsatisfactory and a joint prosthesis is not yet indicated. The potentially negative effect of irrigation fluids on cartilage metabolism and structure has led to the development of a temporary synovial fluid substitute containing hyaluronic acid. The short and long-term effects of this synovial fluid substitute were investigated in a total of 80 patients with persistent knee pain. Forty patients underwent arthroscopic knee joint lavage, in some cases combined with careful cartilage debridement (group A) while a further 40 patients underwent the same procedure which, after final joint lavage, was immediately followed by a single instillation of $10 \mathrm{ml}$ of the synovial fluid substitute ( $0.5 \%$ sodium hyaluronate) into the joint (A + HA group). After the procedure, pain on walking and restricted ability to walk $100 \mathrm{~m}$ were markedly reduced to a comparable extent in both groups. Three months later, the effect of the treatment assessed using various parameters (CGI, restricted ability to walk $100 \mathrm{~m}$, pain on walking, night pain) had decreased in group A, while it remained stable or even improved slightly in the A + HA group. The MannWhitney statistics revealed a descriptive superiority for the A + HA group at this time point. One year after treatment the superiority of the A + HA group was confirmed using the same assessment parameters. No side effects or adverse events were observed for either treatment procedure. This study shows that arthro-
\end{abstract}

H. Hempfling $(\bowtie)$

Berufsgenossenschaftliche Unfallklinik,

Prof.-Küntscher-Strasse 8,

82418 Murnau am Staffelsee, Germany

e-mail: hempfling@bgu-murnau.de scopic knee joint lavage leads to a lasting improvement in pain and functional impairment. The post-arthroscopic instillation of a HA-based synovial fluid substitute into the joint is a suitable way of achieving long-term stabilisation of the treatment outcome. This was supported by findings of a survey of 66 patients at 2 years after treatment in this study. Level I prospective, randomised controlled double-blind study.

Keywords Intra-articular - Hyaluronic acid .

Knee osteoarthritis - Arthroscopic knee joint lavage . Debridement

\section{Introduction}

Pain and impaired knee joint function reduces the radius of action and quality of life of the patients concerned. Osteoarthritic changes play a causative role particularly in the elderly [1]. Where conservative measures such as physiotherapy, oral analgesics, nonsteroidal anti-inflammatory drugs or intra-articular injections of, for example, corticosteroids are no longer sufficiently effective, arthroscopic knee joint lavage, possibly supplemented by smoothing of the cartilage, may be considered as the next step. The purpose of this minimal procedure is to remove the products of cartilage wear, inflammatory cells and molecules from the joint and therefore counter the onset of painful inflammatory phases ("activated osteoarthritis").

Hyaluronic acid (HA) is a long-chain biopolymer made up of continuously repeated molecular sequences of $N$-acetyl-glucosamine and glucuronic acid. In joint cartilage, HA functions as the backbone for proteoglycans, which together with collagen form the extra- 
cellular matrix in which the chondrocytes are embedded. The surfaces of the cartilage and synovial membrane are coated with HA. HA also plays a particular role in synovial fluid, giving it its shock absorbing and viscoelastic properties. In addition, the tightly interwoven macromolecules of HA in the joint space form a molecular sieve which prevents the free passage of inflammatory cells and molecules, while allowing the flow of catabolic compounds from the synovial membrane to the non-vascularised joint cartilage. In osteoarthritis, exogenous HA injected into the joint space has been shown to improve the qualitative and quantitative properties of endogenous HA and therefore improve joint lubrication (concept of viscosupplementation) [1].

During arthroscopy, the irrigation fluid not only removes harmful detritus but also the synovial fluid and the HA layer covering the joint tissues including the cartilage. Although most of the fluid is removed from the joint after the procedure, some remains and experimental studies have shown that irrigation fluids used in arthroscopy can have a negative effect on the metabolism and structure of the joint cartilage [2, 18, 20]. This led to the development of the concept of replacing the lost synovial fluid with an HA-containing synovial fluid substitute immediately after arthroscopy. The aim of this study was therefore to investigate the short- and long-term effects and safety of such an HAcontaining synovial fluid substitute when used after arthroscopic joint lavage.

\section{Patients and methods}

A total of 80 patients suffering from severe knee joint pain for at least 6 months (pain VAS $>50 \mathrm{~mm}$ ) and in whom arthroscopic joint lavage (and possibly intraoperative cartilage smoothing) was indicated were recruited into a single-centre, randomised, controlled, double blind study. The study protocol was approved by an Institutional Review Board (IRB) and the informed consent was obtained from each patient. The study was conducted in accordance with the study protocol and good clinical practice (GCP) [11]. Patients with joint disease that could interfere with the outcome of treatment, those with severe systemic disease, those in whom the investigational product or arthroscopic treatment were contraindicated and those who had received intra-articular treatment during the 6 months preceding the study were excluded from the study. The use of other therapeutic agents was prohibited throughout the study, except for pain medication (acetaminophen up to $2 \mathrm{~g}$ per day). During the first 2 weeks following the procedure an accurate record was to be kept of any acetaminophen taken.

All 80 patients were treated with the same arthroscopic technique, as follows. Patients were positioned on their backs with the knee to be treated supported by a knee wedge in approximately $30^{\circ}$ flexion. A tourniquet cuff was fitted as a precautionary measure. The procedure was carried out under general anaesthesia, nerve block and, in a few cases, local anaesthesia. Once anaesthesia was established, the arthroscope $(5.5 \mathrm{~mm}$ external diameter) was introduced through a $3-4 \mathrm{~mm}$ stab incision using the anterolateral or high anterolateral approach.

First a thorough examination of the entire joint was carried out with precise location of the chondral lesion and its grading according to the Outerbridge classification [17] The chondral lesions were then treated by lavage or debridement. Lavage involved irrigating the knee joint with at least 3,000 ml Ringer's solution while debridement involved the smoothing of the cartilage and/or meniscus. All smoothing procedures were performed with restraint and, where possible, only necrotic or floating chondral tissue was removed from the cartilage, creating mechanically favourable transitions with the adjacent chondral surfaces. The meniscus was resected only where there were degenerative tears to the posterior horn and concomitant degenerative chondral lesions. In this case the torn section of meniscus was resected and the margin carefully smoothened. For debridement, rongeurs and chisels were used for the removal of exophytes. Occasionally shaver systems were also used, always combined with subsequent lavage. The irrigation pressure was sufficiently high to produce a jet of fluid containing detritus and tissue fragments through the arthroscope shaft. Suction drains were not required in any patient and there was no postoperative resting, so that the earliest possible mobilisation with full weight bearing was achieved. No specific treatment was prescribed during the complete follow-up period.

Forty patients received only arthroscopic treatment (group A) while a further 40 patients received an instillation into the joint of $10 \mathrm{ml}$ of a $0.5 \%$ HA solution (Viscoseal ${ }^{\circledR}, 10 \mathrm{ml} / 50 \mathrm{mg}$ ampoules, TRB Chemedica AG, D-85540 Haar) after completion of the procedure and removal of the irrigation fluid (group A + HA). The concentration of HA used in Viscoseal ${ }^{\circledR}$ is the same as that of healthy synovial fluid, with a mean molecular weight of 1.6 million Daltons. Viscoseal $^{\circledR}$ is presented in flexible ampoules which are sealed into a sterile sachet and then autoclaved. The double blind nature of the study was ensured as follows: the patient was either under general anaesthesia 
throughout the treatment or, where epidural or local anaesthesia was used, had no view of the operating area. The doctor making the assessments was given no information on the type of treatment each patient received. The status of the joint prior to treatment was assessed during the operation using the Outerbridge classification of chondral lesions [17]. The medial and lateral femoral condyle, the medial and lateral tibial plateau and medial and lateral patella plus trochlea were assessed using a four-point score $(1=$ local softening and swelling of the cartilage, 2 = fissure formation and fragmentation up to a diameter of $1.3 \mathrm{~cm}$, 3 = fissure formation and fragmentation with a diameter of more than $1.3 \mathrm{~cm}, 4=$ ulcers and erosions reaching the subchondral bone).

The efficacy of the treatment was assessed using validated scales and patient questionnaires. The primary efficacy parameter was pain monitored using the Huskisson $100 \mathrm{~mm}$ visual analogue scale (VAS) [10] on which 0 indicates "no pain" while 100 indicates "unbearable pain". Pain assessments were performed both, by the patients through daily entries in a pain diary for 2 weeks postoperatively and by the Investigator at $6 \mathrm{~h}$ and 2 weeks after the operation. The patient's overall impression of the treatment outcome (clinical global impression, CGI) was assessed using a five-point ordinal scale $(1=$ very good/symptom-free, $2=\operatorname{good} /$ clearly improved, $3=$ satisfactory/slightly improved, $4=$ unchanged, $5=$ worse). Restricted ability to walk $100 \mathrm{~m}$ were recorded using a five-point ordinal scale $(0=$ no difficulties, $1=$ slight, $2=$ considerable, $3=$ major, $4=$ extreme). The presence/absence of pain on walking $100 \mathrm{~m}$ (pain on exercise) and in the night (pain at rest) were recorded (Yes/No). Assessments were made at baseline ( $\mathrm{V} 0$-i.e. 1-2 days before the operation), at time of operation (V1-i.e intraoperative findings), and then at $6 \mathrm{~h}$ (V2), 2 weeks (V3), 3 months (V4) and 1 year (V5) post-surgery (Tables 1 ). This timescale allowed the assessment of the short-
(V2, V3 and V4) and long-term (V5) effects of treatment. To assess the longer-term outcome, patients completed a further questionnaire at 2 years postsurgery. This recorded CGI, pain on walking $100 \mathrm{~m}$, night pain and restricted ability to walk $100 \mathrm{~m}$.

\section{Statistical evaluation}

Since joint irrigation is described in the literature as an effective treatment for knee problems, we wished to determine whether non-inferiority could be demonstrated for the investigational product, Viscoseal ${ }^{\circledR}$.

Data for all patients were used in evaluating the safety aspect of the investigational product. Thirtyeight patients from group $\mathrm{A}$ and 36 patients from the A + HA group were included in the ITT (intention-totreat) data set for primary analysis. Statistical evaluation initially examined whether the new treatment method A + HA was not inferior to the established procedure A with regard to efficacy. If significance was achieved, a test for superiority was performed at the same alpha level $(2.5 \%)$. The hypotheses were tested using the measure of relevance. In the case of the primary efficacy parameter (pain VAS), standardised difference $(=\mathrm{diff} / \mathrm{s})$ was used, while the secondary parameters were analysed using the Mann-Whitney reference value. The limit for non-inferiority was the mean inferiority according to Cohen and the limit for superiority was equality according to Cohen. The findings of the patient survey 2 years after the procedure were also subjected to statistical evaluation as described (Table 2).

\section{Results}

A total of 41 women and 39 men (mean age $60.9 \pm 8.1$ years; mean height $171.2 \pm 8.5 \mathrm{~cm}$; mean

Table 1 Study timetable

\begin{tabular}{|c|c|c|c|c|c|c|}
\hline $\begin{array}{l}\text { Pre } \\
\text { (V0) }\end{array}$ & $\begin{array}{l}\text { Intra-op } \\
\text { (V1) }\end{array}$ & $\begin{array}{l}6 \mathrm{~h} \text { post } \\
(\mathrm{V} 2)\end{array}$ & $\begin{array}{l}2 \text { Weeks } \\
\text { (V3) }\end{array}$ & $\begin{array}{l}3 \text { Months } \\
\text { (V4) }\end{array}$ & $\begin{array}{l}1 \text { Year } \\
\text { (V5) }\end{array}$ & $\begin{array}{l}2 \text { Years } \\
\text { (V6) }\end{array}$ \\
\hline
\end{tabular}

Findings on inclusion, inclusion and exclusion $\mathrm{X}$ criteria

Outerbridge assessment

VAS (assessed by Investigator)

VAS pain diary (patient)

Clinical global impression, patient (CGI)

Restricted ability to walk $100 \mathrm{~m}$

Pain on walking $100 \mathrm{~m}$

Night pain

Safety

\begin{tabular}{clc}
\multicolumn{1}{c}{$\mathrm{X}$} & & \\
$\mathrm{X}$ & $\mathrm{X}$ & $\mathrm{X}$ \\
& \multicolumn{2}{c}{ Daily } \\
& $\mathrm{X}$ & $\mathrm{X}$ \\
$\mathrm{X}$ & $\mathrm{X}$ & $\mathrm{X}$ \\
$\mathrm{X}$ & $\mathrm{X}$ & $\mathrm{X}$ \\
$\mathrm{X}$ & $\mathrm{X}$ & $\mathrm{X}$ \\
& $\mathrm{X}$ & $\mathrm{X}$
\end{tabular}

$\begin{array}{lll}\text { X } & \text { X } & \text { X } \\ \text { X } & \text { X } & \text { X } \\ \text { X } & \text { X } & \text { X } \\ \text { X } & \text { X } & \text { X } \\ \text { X } & \text { X } & \text { X }\end{array}$


Table 2 Intra-operative assessment of chondral lesions per compartment according to Ficat and Hungerford (number of patients affected)

\begin{tabular}{llcc}
\hline & $\begin{array}{l}\text { Medial } \\
\text { compartment }\end{array}$ & $\begin{array}{l}\text { Lateral } \\
\text { compartment }\end{array}$ & $\begin{array}{l}\text { Patellofemoral } \\
\text { compartment }\end{array}$ \\
\hline A group & & & \\
Grade 1 & 1 & 6 & 3 \\
Grade 2 & 24 & 16 & 27 \\
Grade 3 & 14 & 4 & 4 \\
A + HA group & & 2 \\
Grade 1 & 3 & 3 & 23 \\
Grade 2 & 22 & 18 & 8 \\
Grade 3 & 14 & 9 & \\
\hline
\end{tabular}

body weight $79.6 \pm 14.7 \mathrm{~kg}$ ) were included in the study. The comparison of homogeneity for these and almost all other parameters at the baseline visit (V0) showed equality between the two groups of patients. Slight differences between the groups were found only with respect to restricted ability to walk $100 \mathrm{~m}$ (Fig. 3).

To allow better comparability with other studies, the findings of the intra-operative assessment (V1) of chondral lesions, according to the four-stage Outerbridge scheme, were transferred to the three-stage classification of Ficat and Hungerford [6]. This was achieved by combining Outerbridge stages II and III into Ficat and Hungerford stage II. In this assessment there was found to be slightly more severe damage to the medial (group A $2.16 \pm 0.52$; group A $+\mathrm{HA}$ $2.23 \pm 0.59$ ) and patellofemoral compartments (group A $2.02 \pm 0.51$; group A + HA $2.17 \pm 0.55$ ) compared to the lateral joint compartment (group A $1.86 \pm 0.61$; group $\mathrm{A}+\mathrm{HA} 2.03 \pm 0.65)$ and more pronounced chondral lesions in group A + HA than in group A. No correlation was found between the location and severity of chondral damage and the preoperative clinical findings or the postoperative course. No appreciable damages of the menisci were found in the study population. In light of the arthroscopic findings, cartilage debridement with joint lavage was performed in 9 patients in group $A$ and 11 patients in group A + HA. As the postoperative progress of patients who also underwent debridement did not differ appreciably from that of patients who underwent joint lavage alone, these patients were also included for subsequent assessments.

Changes in pain symptoms (VAS) during the 14 days post-surgery (V0, V2 and V3) were very positively assessed both by the doctor and by the patients. Assessment by the doctor showed that the previously severe pain decreased markedly in both groups as a result of arthroscopic treatment of the joint. In the group not receiving subsequent treatment with HA (group A) the severity of the pain showed a mean decrease from $66.1 \pm 11.7 \mathrm{~mm}$ VAS before the procedure to $20.2 \pm 18.0 \mathrm{~mm}$ at $6 \mathrm{~h}$ after the procedure and to $17.1 \pm 20.1 \mathrm{~mm}$ at 14 days. In the A + HA group, pain decreased in a similar way from $65.8 \pm 10.4 \mathrm{~mm}$ (V0) to $20.5 \pm 16.4 \mathrm{~mm}$ (V2) and $15.8 \pm 13.8 \mathrm{~mm}$ (V3). Daily records made by the patients showed a decrease in pain severity by $40.8 \%$ by Day 14 in group A and $42.7 \%$ in group A + HA. An inter-group comparison showed no significant difference between the groups.

During the interval between V4 and V5, two patients from group $\mathrm{A}$ and four from group $\mathrm{A}+\mathrm{HA}$ left the study for reasons (joint replacement, repeat arthroscopy required) not attributable to study treatment. Therefore, 1 year after the procedure, data for 74 patients (group A 38, group A + HS 36) were still available for assessment of CGI, pain during exercise and at rest and restricted ability to walk $100 \mathrm{~m}$.

The overall impression of patients regarding the success of the treatment (CGI) was very positive in both groups over a period directly following the procedure but subsequently showed increasing superiority of the A + HA group up to 1 year afterwards (Fig. 1). Shortly after the procedure (V2) most patients reported a marked improvement or were even symptomfree $(87.5 \%$ in the A group, $90.0 \%$ in the A + HA group). This assessment remained largely unchanged after 14 days (V3) $(82.5 \%$ in the A group, $87.5 \%$ in the A + HA group). Three months after arthroscopy (V4), however, the treatment outcome was assessed to be considerably more favourable in the A + HA group ("clearly improved" and "free from symptoms": $82.5 \%$ compared with $65.0 \%$ in the A group). This trend was even more pronounced 1 year after treatment (V5). Seventy-five percentage of patients from the A + HA group had a good to very good global impression of the treatment while this was the case in only $50 \%$ of patients from the A group. In the statistical analysis (Mann-Whitney), this trend was reflected as follows: at 14 days (V3) and 3 months (V4) after arthroscopy, non-inferiority was recorded for the A + HA group, whereas 1 year after the procedure (V5) proven superiority was observed for the A + HA group (Fig. 2).

Before arthroscopy, $76.9 \%$ of patients from the A group and $85.0 \%$ from the A + HA group (Fig. 4) complained of restricted ability to walk $100 \mathrm{~m}$. Compared with the baseline findings, over $50 \%$ of the patients in the A group showed an improvement of one or more score points, (e.g. from "extreme" to "slight" difficulties (V2 59.0\%, V3 61.5\%, V4 59.0\%, V5 $59.5 \%$ ) concerning this parameter. However, the number of improved patients was considerably higher 
Fig. 1 Clinical global impression (CGI) by the patients during the study. (A group arthroscopy;

A + HA group arthroscopy + hyaluronic acid)
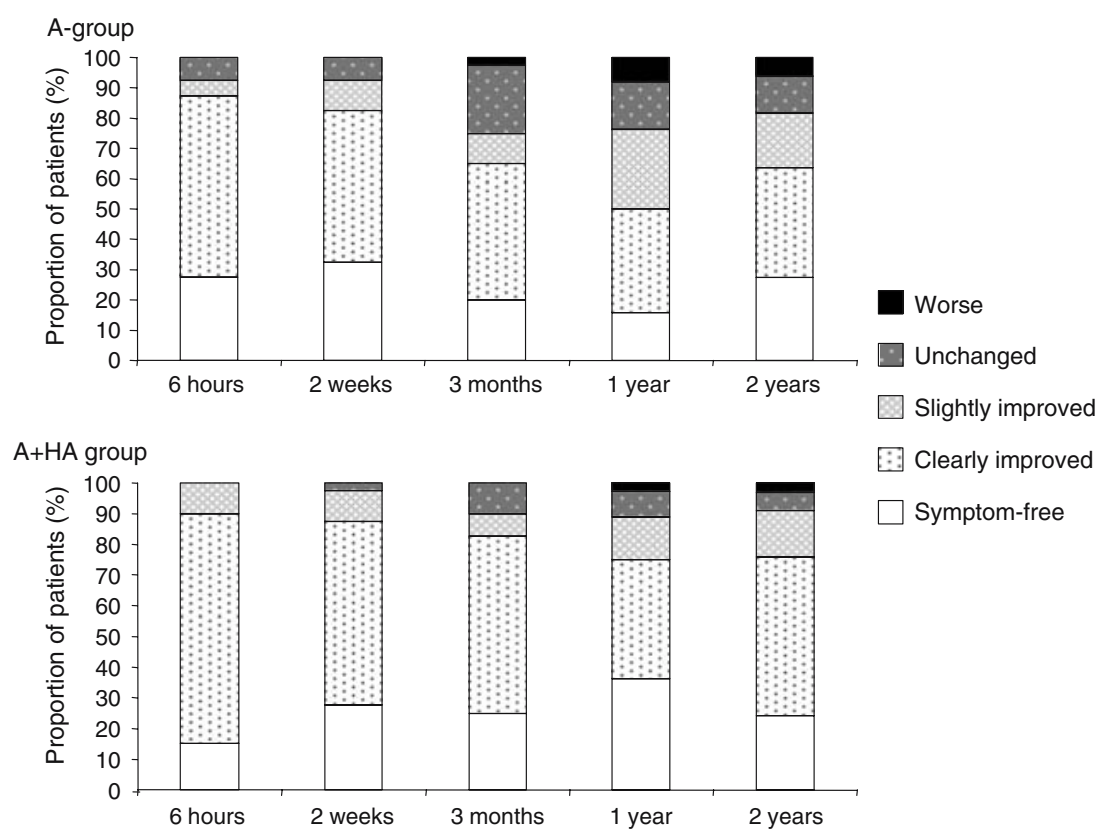

Clearly improved

Symptom-free

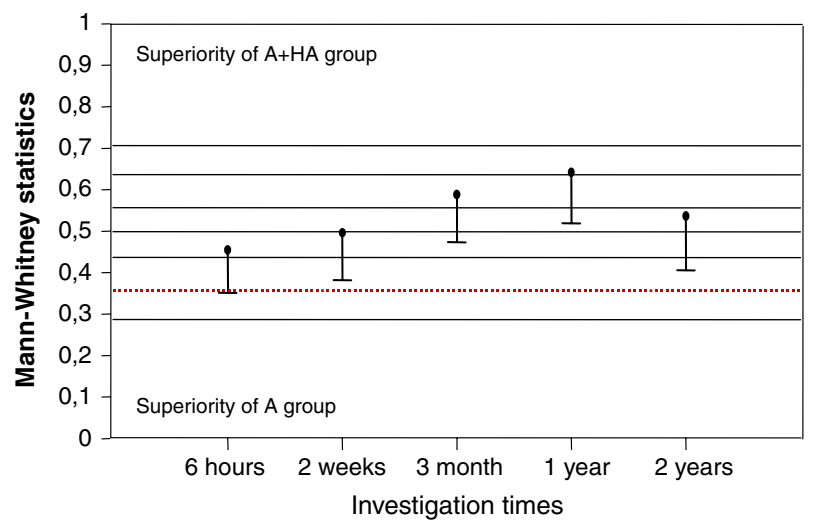

Fig. 2 Clinical global impression by patients. The dashed line at 0.36 is the benchmark for the effect size 'medium sized inferiority'. At 3 months after treatment the Mann-Whitney statistics showed a descriptive superiority in favour of the A + HA group while at 1 year after treatment there was a proven superiority (since Mann-Whitney value and the confidence interval are above the benchmark for 'equality' at 0.5 ) in favour of the A + HA group. The Mann-Whitney statistics for all other efficacy parameters led to similar results. (A group arthroscopy; A + HA group arthroscopy + hyaluronic acid)

in the $\mathrm{A}+\mathrm{HA}$ group (V2 $75.0 \%, \mathrm{~V} 377.5 \%$, V4 $77.5 \%$, V5 $77.8 \%$ ). The number of patients with no restricted ability to walk increased in group A from $23.1 \%$ preoperatively to $60.5 \%$ at 1 year (V5) after the procedure. In the A + HA group a very much clearer increase in the percentage of symptom-free patients, from 15.0 to $88.9 \%$, was observed. Also, at the last visit 9.1\% of the patients in group A still had "major" problems in walking $100 \mathrm{~m}$, while none of the patients from the A + HA group reported more than "considerable" problems (Fig. 3). Statistical analysis (MannWhitney), as with the global assessment of efficacy by patients, again showed non-inferiority at 14 days (V3) and 3 months (V4) after the procedure and proven superiority at 1 year (V5). These data are not presented here.

Before arthroscopy, $76.9 \%$ of the patients from group $\mathrm{A}$ and $87.5 \%$ from the $\mathrm{A}+\mathrm{HA}$ group complained of pain on walking $100 \mathrm{~m}$ (Fig. 4). Immediately after the arthroscopy, considerably fewer patients in both groups initially suffered pain on walking (V2 group A $32.5 \%$, A + HA group $41.0 \%$ ). During the study however, the proportion of pain-free patients in group A increased only slightly and after 1 year (V5) more than one-third of the patients (39.5\%) still complained of pain on walking. In contrast, the proportion of pain-free patients in the A + HA group increased continuously during the study such that at 1 year (V5) only $13.9 \%$ still complained of pain on walking. Statistical analysis (Mann-Whitney) again showed non-inferiority for the A + HA group at 14 days (V3) and 3 months (V4) and proven superiority at 1 year (V5) after the procedure.

Another efficacy parameter in this study was pain at rest ("night pain") (Fig. 5). Before arthroscopy 48.7\% of patients in group A and $66.7 \%$ in the A + HA group had reported this symptom. Immediately after arthroscopy, most patients were initially pain-free (at V2 $92.5 \%$ in group A, $90.0 \%$ in the A + HA group). However the proportion of patients with pain at rest increased continuously in group A: at 1 year (V5) 
Fig. 3 Restricted ability to walk $100 \mathrm{~m}$ (A group arthroscopy; A + HA group arthroscopy + hyaluronic acid)
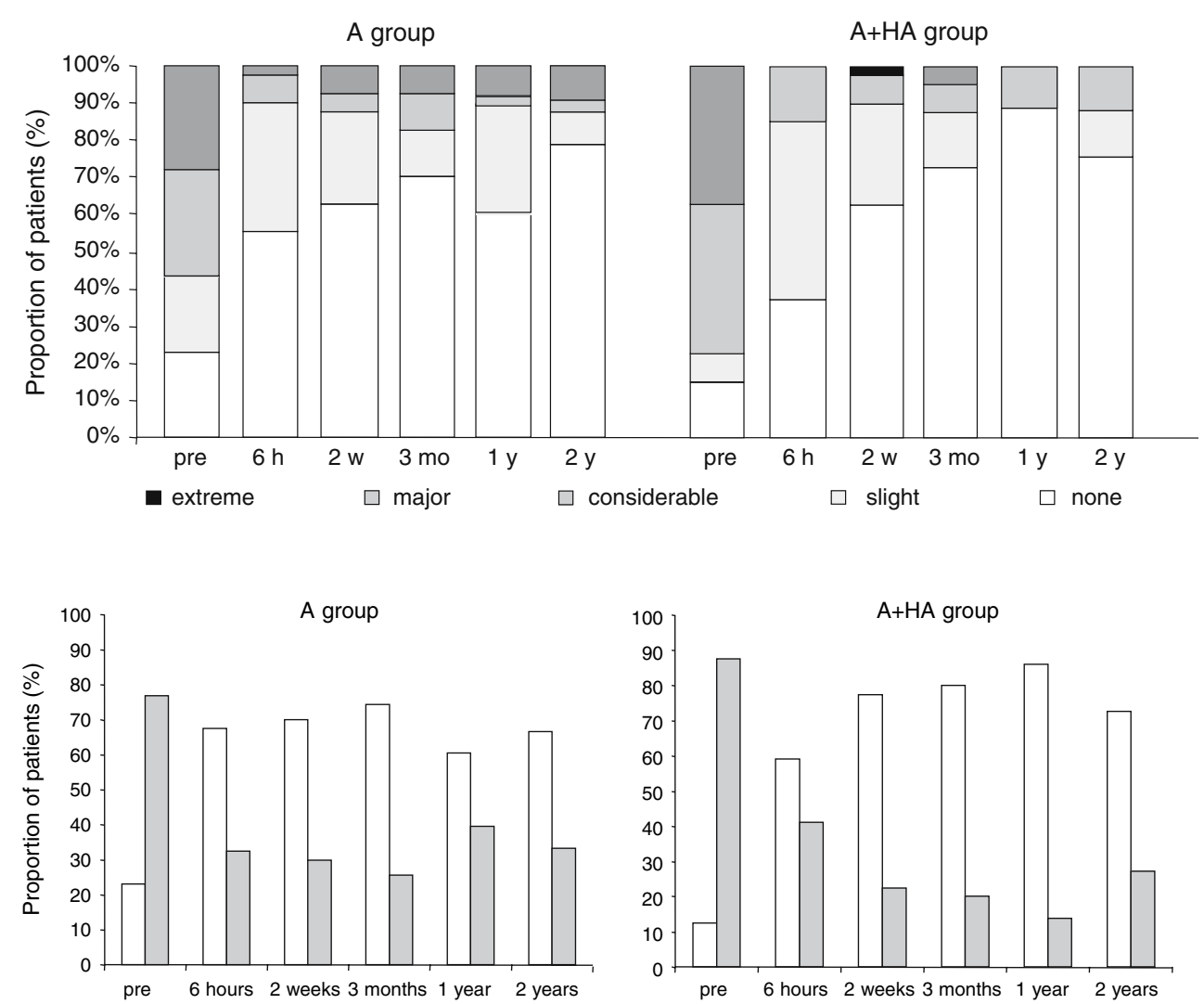

Fig. 4 Pain on walking $100 \mathrm{~m}$. The light bars represent pain-free patients, the dark bars patients with pain. (A group arthroscopy; A + HA group arthroscopy + hyaluronic acid)

Fig. 5 Night pain. The light bars represent pain-free patients, the dark bars patients with pain. (A group arthroscopy; A + HA group arthroscopy + hyaluronic acid)

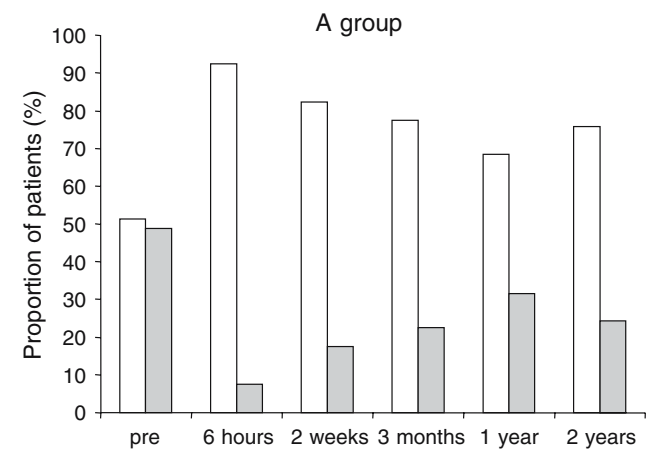

$31.6 \%$ of these patients again reported this symptom. In contrast, only $11.1 \%$ of the patients in the A + HA group complained of pain at rest at this visit. Statistical analysis by the Mann-Whitney method for the parameter pain at rest at $6 \mathrm{~h}$ (V2) after arthroscopy and again at 14 days (V3) and 3 months (V4) showed noninferiority for the A + HA group. One year after the procedure (V5) proven superiority was observed for the A + HA group. Adverse events or effects showing a causal relationship with the investigational product were not recorded in any patient.

Data for 66 patients (33 in each group) were available for statistical assessment at 2 years after the procedure: eight patients had to undergo additional treatment after visit 5 (e.g. total knee replacement, arthroscopy or shock wave treatment) and were therefore not included in the assessment. The mean time to the two-year follow-up was 414.7 days (range 308-559 days) for the patients in group $\mathrm{A}$ and 423.5 days (range $314-560$ days) for the patients in the A + HA group.

Although differences between the groups were by now less clearly pronounced, more patients $(75.8 \%)$ in the A + HA group assessed the treatment outcome as positive than in group A $(63.6 \%)$ (Fig. 1). Similar numbers of patients in the two groups had no restricted ability to walk $100 \mathrm{~m}$ at 2 years (A 78.8\%, A + HA $75.8 \%$ ) (Fig. 3). However, the number of improved 
patients compared with the baseline visit, was clearly greater in the A + HA group (improvement of one or more score points in $75.8 \%$ of patients) than in group A $(56.3 \%)$. At 2 years almost a third of the patients in both groups suffered from pain on walking $100 \mathrm{~m}$ (A $33.3 \%$, A + HA 27.3\%) (Fig. 4). Pain at rest affected only $12.1 \%$ of patients in the group treated with HA compared to twice as many patients $(24.2 \%)$ from group A (Fig. 5). The Mann-Whitney statistics showed a descriptive superiority for the A + HA group for the global assessment by patients, pain on walking $100 \mathrm{~m}$ and pain at rest, while non-inferiority was shown for the A + HA group for restricted ability to walk $100 \mathrm{~m}$. There were no signs of any undesirable effects due to Viscoseal ${ }^{\circledR}$

In summary, it can be stated that there was no difference between the two treatment methods in their short-term effect. However, the temporary replacement of synovial fluid with Viscoseal ${ }^{\circledR}$ after arthroscopy conferred additional benefits for a considerable proportion of the patients treated in this way. Findings at 1 year for all efficacy parameters were more favourable in this group of patients and the MannWhitney statistics consistently demonstrated the superiority of this treatment regime. Findings from the patient follow-up at 2 years also showed that Viscoseal $^{\circledR}$ still had a stabilising effect on the beneficial effects of lavage and debridement. No adverse events or effects of the supplementary treatment with HA were found in any patient and therefore the benefit-risk assessment of synovial fluid substitution with Viscoseal ${ }^{\circledR}$ was positive overall.

\section{Discussion}

The treatment of osteoarthritic changes with arthroscopic joint lavage, possibly supplemented by cartilage smoothing, may be used when conservative measures are no longer sufficiently effective. The underlying assumption is that the deposition of small particles of worn cartilage in the synovial membrane causes a nonbacterial form of synovitis and an associated increase in effusion leading to continuing pain and irritation of the joint ("activated osteoarthritis"). Irrigation not only removes the particles of worn cartilage (detritus) but also the enzymes that destroy cartilage. Additional debridement removes fragments of cartilage that are loosened but still attached, from which further detritus could arise. The surgically removed fragments must also be thoroughly washed out of the joint.

Literature reports at 2 years show that $25-93 \%$ (average $61.0 \%$ ) of patients undergoing joint lavage alone and between 0 and $94 \%$ (average $62.5 \%$ ) of those also undergoing debridement had improvements in their symptoms [8]. Later publications by Muckley [16] and Hempfling [9] compared the carry-over effects of knee joint lavage with those of joint lavage + debridement over a period of 4 years after the procedure. It was found that the debridement group benefited more in the three-year period after the procedure than those in whom only joint lavage was carried out. Four years after the procedure, patients assessed the two procedures as almost equally effective. In view of the favourable course during the first 3 years, sparing debridement is therefore preferable to joint lavage alone. In their studies Muckley and Hempfling also investigated the effect of the severity of chondral lesions, assessed according to Ficat and Hungerford, on the postoperative outcome. They found that the greater the severity of chondral damage, the less good the outcomes were in the period up to 4 years after the procedure. Patients with low-grade damage showed a considerably more pronounced improvement which persisted for a longer period. If progress (using the CGI parameter) at 2 years from the studies mentioned above is compared with the present study, it is again clear that patients treated with HA derive additional benefit: this applies both for the assessment according to treatment (i.e. lavage alone versus lavage + debridement) and also according to the severity of arthroscopically classified chondral lesions (Figs. 6, 7).

In contrast with the above findings, a prospective controlled study by Moseley et al. [15] in patients with osteoarthritis of the knee found no appreciable differences between the results of joint lavage alone,

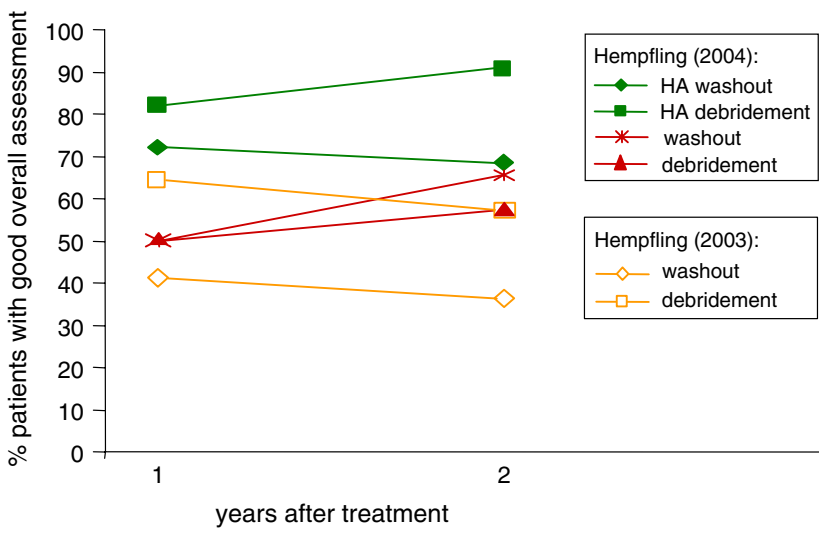

Fig. 6 Overall assessment by patients after joint lavage or joint lavage plus debridement. Patients with the assessment "very good" and "good" for the CGI were taken together as patients with a good overall result. The patient groups treated with hyaluronic acid are marked with HA 


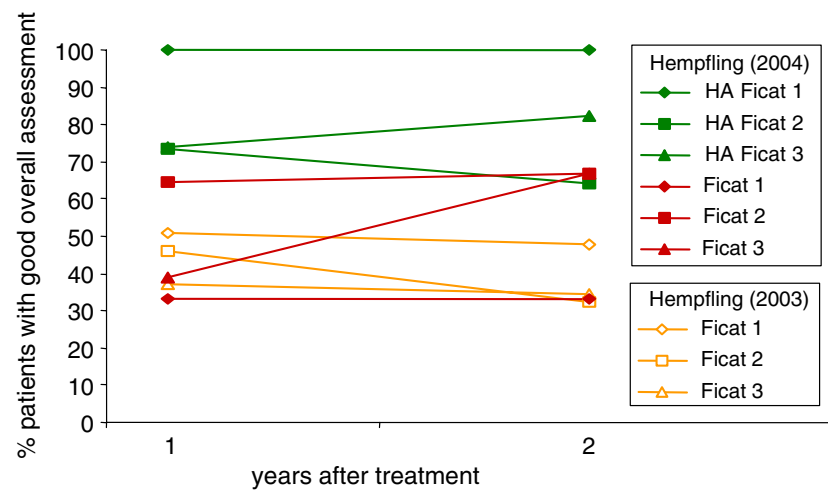

Fig. 7 Overall assessment by patients after joint lavage or joint lavage plus debridement related to the severity of chondral damage before treatment

debridement and a placebo operation. In this study, severity was radiologically classified according to Kellgren [12]. Precise arthroscopic classification of the intra-articular status (e.g. according to Outerbridge or Ficat and Hungerford) was not carried out-particularly in the placebo group which received only a skin incision. Torn meniscus was resected not only in the debridement group but also in some cases in the lavage group. Radiological classification of chondral lesions is not comparable with arthroscopic classification, since some lesions that can be detected by arthroscopy cannot be visualised radiologically. Moseley et al. [15] evidently detected only those chondral lesions that had already resulted in radiologically detectable secondary damage. Comparison of the results described here with those of Moseley et al. is therefore not possible.

The positive result of both joint lavage alone and of joint lavage combined with debridement is a joint free from detritus. However, during joint lavage the synovial fluid is also completely washed out of the joint. In experimental studies, irrigating fluids have been shown to have a negative effect on cartilage metabolism. Bulstra et al. [3] carried out in-vitro investigations on the rate of proteoglycan synthesis under the influence of various irrigating fluids on complete rat patella explants. The preparations were incubated for $1 \mathrm{~h}$ in each solution and the rate of proteoglycan synthesis determined for a period of $16 \mathrm{~h}$ based on ${ }^{35} \mathrm{SO}_{4}$ incorporation into the cartilage. All irrigation fluids inhibited the rate of proteoglycan synthesis. Negative deviation from the control was $5 \%$ for Ringer's solution, $10 \%$ for Ringer's glucose, $20 \%$ for physiological saline and Ringer's lactate and $55 \%$ for Betadine. In another study, Reagan et al. [18] placed sections of cartilage in various commercially obtainable irrigation fluids. ${ }^{35} \mathrm{SO}_{4}$ incorporation showed that physiological saline and phosphate buffer, compared with normal nutrient media, had a negative effect on cartilage metabolism. Similar findings were also obtained by Straehley et al. [20].

Several studies have been carried out to investigate the effects of using HA-containing solutions as a synovial fluid replacement following arthroscopy and also to remove potentially harmful irrigating solutions from the joint. The first investigations were performed 1995 by Edelson et al. [4] in 26 patients ( 33 knees) with symptoms refractory to treatment due to radiologically confirmed degenerative disease of the knee joint. All knees underwent joint lavage and 16 were then given an intra-articular injection of $3 \mathrm{ml} \mathrm{HA}$ solution (molecular weight of HA: $0.5-1$ million Da). All patients experienced an improvement in their symptoms (pain and function) which was maintained for up to 2 years. There was no significant difference between the two methods of treatment. Unfortunately, the report gives no indication of adequate biostatic study planning or of the characteristics and amount of the HA administered, which reduces the significance of the study. In a randomised study in patients with osteoarthritis of the knee, Foster and Straw [7] compared the effect of arthroscopic irrigation with a series of five intra-articular (i.a.) injections of extractive HA. After aspiration of the synovial fluid, 19 patients received i.a. HA injections, while 17 patients underwent joint lavage with physiological saline, sometimes combined with debridement. At baseline, the Knee Society Function Score in patients in the arthroscopy group showed significantly worse values. At the end of the study, inter-group comparison for all parameters investigated showed the superiority of HA. However, the extent of the improvement was not reported. Moreover, 12 patients dropped out during this oneyear study of which 10 were from the HA group. Five of these HA patients required arthroscopic treatment and three were referred for knee replacement. It is therefore possible that at the end of the study the HA group consisted only of those nine patients who remained in the study because of satisfactory treatment outcomes. In this case the statistical conclusion must be considered with great caution.

The benefit of the combined use of joint irrigation and i.a. HA was analysed by Vad et al. [21] in a study of 81 patients with osteoarthritis of the knee. All patients were treated with three i.a. injections of crosslinked extractive $\mathrm{HA}$ at weekly intervals. In 44 patients, joint lavage was performed using the closed needle method 1 week before starting the series of injections. Patients who obtained pain reduction of at least $50 \%$ and those who assessed treatment outcome as good to excellent were judged to have been suc- 
cessfully treated. According to this criterion, the success rate for i.a. HA alone was $54 \%$, at 1.1 years after treatment while it was $79.5 \%$ for the combined treatment comprising lavage and HA. Moreover, eight patients reacted to the treatment with local reddening and pain, which resolved spontaneously in only six patients. Mathies [14] investigated the effects of temporary replacement of synovial fluid with a $0.5 \% \mathrm{HA}$ solution (Viscoseal ${ }^{\circledR}($ ) after arthroscopic meniscectomy. A suction drain was installed for postoperative patient care in all the 50 patients included in the study. In 25 patients the HA solution was instilled into the joint through the suction drain which was then blocked and the knee manipulated. The drain was reopened 2030 min later on the assumption that sufficient HA would by then have been deposited on the joint surfaces. Pain at rest and on exercise was considerably less in patients in the HA group during the first few days after the procedure. In fact, at the final examination on day 28, patients in the HA group showed a much more pronounced reduction in joint swelling. They also took less diclofenac during the study, had better Lysholm scores and assessed the outcome of the procedure more favourably overall. In the Investigator's view, the benefit of Viscoseal ${ }^{\circledR}$ administration was that patients could be mobilised earlier because of reduced pain. The further postoperative progress of the patients in this study is not reported.

The acute effects of exogenous HA in the joint (for example, improved lubrication) are evident and the subject of many publications. The mechanisms underlying the carry-over-effect are not yet well understood. Bulstra [3] in a rabbit model of chronic osteoarthritis, showed that intra-articular HA following joint lavage was able to enhance the chondrocyte metabolism and reduce the rate of chondrocyte apoptosis. Smith and Ghosh [19] in cultures of "arthritic" human synoviocytes, showed that exogenous HA stimulated the synthesis of endogenous HA. Only recently, in patients who fulfilled the criteria for knee replacement, Mathies et al. [13] reported a long lasting improvement of the viscous and elastic moduli of their synovial fluid after five intra-articular injections of HA, which was paralleled by a clinical improvement such that most patients did not require arthroplasty during the 12 months after treatment.

In our current study, the short-term benefit of postarthroscopic substitution of synovial fluid with HA described by Mathies et al. [14] was not demonstrated. It may be that the more severe pain symptoms due to the placing of the suction drain played a role. In our two-year study, however, the positive long-term effects of this new treatment option were robustly demon- strated over all efficacy parameters. The stabilising effect of Viscoseal ${ }^{\circledR}$ on the clinical improvement achieved by lavage and debridement was still evident even 2 years after treatment.

The positive result of the present study is further supported by direct comparison with the findings of Hempfling [9] (Fig. 6). If the assessment by patients in the HA group of this study is compared with that of the group of patients not treated with HA from the current and previous studies, the additional benefit of the HA treatment becomes very evident. It is interesting that the findings for patients treated with $\mathrm{HA}$ in the debridement group are better than those for irrigation alone. The effect of the severity of chondral damage was also taken into account, patients receiving irrigation alone and irrigation + debridement being taken together for a more easy understanding (Fig. 7). Though the groups of patients being considered may be different, and a direct comparison of the data from previous studies with those from the present study must be interpreted with caution, it seems that HA treatment led to better results over 2 years for all levels of severity.

In summary, it may be said that the use of Viscoseal ${ }^{\circledR}$ after arthroscopy is beneficial both for patients in the lavage group and for those who also underwent debridement. The improvement achieved in function and pain is clearly greater and persists over a period of 2 years. It is interesting to note that particular patients with advanced chondral damage benefit from the use of HA as they still assess the outcome of treatment as being positive 2 years after the procedure. Our study findings document the benefits of treating osteoarthritis of the knee by joint lavage and by joint lavage combined with sparing debridement. If these measures are supplemented at the end of the surgical procedure by intra-articular instillation of $\mathrm{HA}$, even more favourable and longer lasting treatment benefits can be expected, with a clear improvement in the quality of life and mobility of these patients.

\section{References}

1. Balazs EA, Denlinger JL (1993) Viscosupplementation: a new concept in the treatment of osteoarthritis. J Rheumatol 20(Suppl 39):3-9

2. Bulstra SK, Kuijer R, Eerdmans P, van der Linden AJ (1994) The effect in vitro of irrigating solutions on intact rat articular cartilage. J Bone Joint Surg 76B:468-470

3. Bulstra S, Kuijer R, Jansen E, Douw C (2000) The effect of hyaluronan on osteo-arthritic chondrocyte metabolism in the rabbit. OARSI, Barcelona

4. Edelson R, Burks RT, Bloebaum RD (1995) Short-term effects of knee washout for osteoarthritis. Am J Sports Med 23:345-349 
5. Engelhardt M (2003) Epidemiologie der Arthrose in Westeuropa. Dtsch Z Sport 54:171-175

6. Ficat RP, Hungerford DS (1977) Disorders of the patellofemoral joint. Masson, Paris, pp 123-148

7. Foster JC, Straw R (2002) A prospective randomised trial comparing intraarticular sodium hyaluronate injection and arthroscopic washout for knee osteoarthritis. In: 10th congress European society of sports traumatology, knee surgery and arthroscopy, Rome 23rd-27th April, book of abstracts

8. Hempfling H (1995) Farbatlas der Arthroskopie großer Gelenke (Teil 2), Springer, Berlin Heidelberg New York

9. Hempfling H (2003) Ergebnisse der Lavage beim Knorpelschaden. In: Jerosch J, Heisel J, Imhoff A.B Fortbildung Orthopädie-Traumatologie 7 Knorpelschaden. Steinkopf Verlag, Darmstadt

10. Huskisson EC (1982) Measurement of pain. J Rheumatol 9:768-769

11. International conference of harmonisation. ICH-Guideline Topic E6: Note for guidance on good clinical practice (CPMP/ICH/135/95)

12. Kellgren JH, Lawrence JS (1957) Radiological assessment of osteo-arthrosis. Ann Rheum Dis 16:494-502

13. Mathies B, Berger J, Siegfried C, Gurny R (2004) Effect of intra-articular sodium hyaluronate (Ostenil) on improving the quality of life and delaying surgery in patients indicated for total knee replacement. An open, pilot, phase III study. In: Symposium of the international cartilage repair society, Gent
14. Mathies B (2005) Effects of Viscoseal, a synovial fluid substitute, on recovery after arthroscopic partial meniscectomy and joint lavage. Knee Surgery, Sports Traumatology, Arthroscopy 10.1007/s00167-005-0625-x

15. Moseley JB, ÓMalley K, Petersen NJ, Menke TJ, Brody BA, Kuydendall DH, Hollingsworth JC, Ashton CM, Wray NP (2002) A controlled trial of arthroscopic surgery for osteoarthritis of the knee. New Engl J Med 347:81-88

16. Muckley TH, Hempfling H (1996) Ergebnisse der arthroskopischen Lavage und des Debridements beim Knorpelschaden. In: Chir. Praxis. München: Hans Marseille Verlag $\mathrm{GmbH}$, 51:659-672

17. Outerbridge RE (1961) The Etiology of Chondromalacia Patellae. J Bone Joint Surg 43-B:752-757

18. Reagan BF, McInerny VK, Treadwell BV, Zarins B, Mankin HJ (1983) Irrigating solutions for arthroscopy. J Bone Joint Surg 65A:629-631

19. Smith MM, Gosh P (1987) The synthesis of hyaluronic acid by human synovial fibroblast is influenced by the nature of the hyaluronate in the extracellular environment. Rheumatol Int 7:113-122

20. Straehely D, Heller A, Solomons C et al (1985) The effect of arthroscopic irrigating solutions on cartilage and synovium. Trans Orthop Res Soc 10:260

21. Vad VB, Bhat AL, Sculco TP, Wickiewicz TL (2003) Management of knee osteoarthritis: knee lavage combined with hylan versus hylan alone. Arch Phys Med Rehabil 85:634637 